\title{
Infantile-onset autosomal recessive nonprogressive cerebellar ataxia
}

INSERM

\section{Source}

INSERM. (1999). Orphanet: an online rare disease and orphan drug data base. Infantileonset autosomal recessive nonprogressive cerebellar ataxia. ORPHA:284332

A rare, genetic, autosomal recessive cerebellar ataxia disease characterized by nonprog ressive cerebellar ataxia, with onset in infancy, manifesting with delayed motor and speech development, gait ataxia, dysmetria, hypotonia, increased deep tendon reflexes, and dysarthria. Additional variable manifestations include moderate nystagmus on lateral gaze, mild spasticity, intention tremor, short stature and pes planus. Brain imaging reveals cerebellar vermis atrophy. 